\title{
Enhancement of ICAM-1 via the JAK2/STAT3 signaling pathway in a rat model of severe acute pancreatitis-associated lung injury
}

\author{
XIAO HAN $^{1 *}$, YUXI WANG ${ }^{2 *}$, HAILONG CHEN $^{1}$, JINGWEN ZHANG $^{1}$, \\ CAIMING XU' ${ }^{1}$, JIAN LI ${ }^{1}$ and MINGYUE LI ${ }^{1}$ \\ ${ }^{1}$ Department of General Surgery, The First Affiliated Hospital of Dalian Medical University, Dalian, Liaoning 116011; \\ ${ }^{2}$ Department of General Surgery, The Second Affiliated Hospital of Dalian Medical University, \\ Dalian, Liaoning 116023, P.R. China
}

Received February 12, 2015; Accepted October 9, 2015

DOI: $10.3892 /$ etm.2016.2988

\begin{abstract}
Acute lung injury (ALI), which is associated with severe acute pancreatitis (SAP), results from damage to the pulmonary microvascular endothelial cells (PMVECs), which in turn leads to high levels of inflammatory cytokines that destroy PMVECs. However, the molecular mechanisms underlying SAP-associated ALI (SAP-ALI) are currently not well understood. Intercellular adhesion molecule-1 (ICAM-1) has been implicated in the persistent migration and accumulation of neutrophils and macrophages, which in turn has been associated with the increased permeability of microvascular endothelial cells. Signal transduction via the Janus kinase-2 (JAK2)/signal transducer and activator of transcription-3 (STAT3) transcription factors has been shown to be involved in inflammation. The present study aimed to investigate the expression levels of ICAM-1 and JAK2/STAT3 signaling components in a rat model of SAP-ALI. SAP was induced in the rat model, and dexamethasone (DEX) was administered to the treatment group. Subsequently, ICAM-1, interleukin (IL)-6, IL-8, tumor necrosis factor (TNF)- $\alpha$, JAK2, STAT3 and nuclear factor (NF) $-\kappa B$ mRNA expression levels were determined using reverse transcription-polymerase chain reaction; ICAM-1 protein expression levels were determined using western blotting; and IL-6, IL-8 and TNF- $\alpha$ levels were measured via an enzyme-linked immunosorbent assay. In addition, an immunohistochemical analysis of ICAM-1, $\mathrm{NF}-\kappa \mathrm{B}, \mathrm{JAK} 2$ and STAT3 was conducted, and the protein
\end{abstract}

Correspondence to: Professor Hailong Chen, Department of General Surgery, The First Affiliated Hospital of Dalian Medical University, 222 Zhongshan Road, Dalian, Liaoning 116011, P.R. China

E-mail: chenhailong2012@aliyun.com

*Contributed equally

Key words: intercellular adhesion molecule-1, Janus kinase-2, signal transducer and activator of transcription-3, acute lung injury, severe acute pancreatitis, rat expression and cell morphology of the lungs in all rats was analyzed. ICAM-1 mRNA and protein expression levels were significantly increased following induction of SAP, and were significantly decreased in the DEX-treated group. Furthermore, treatment with DEX significantly reduced serum expression levels of IL-6, IL-8 and TNF- $\alpha$ and decreased expression levels of NF- $\kappa$ B, JAK2 and STAT3 in the lung tissue, as compared with the untreated SAP group. The present study demonstrated that DEX treatment was able to suppress ICAM-1 mRNA and protein expression in a rat model of SAP-ALI via the inhibition of IL- 6 and TNF- $\alpha$-induced JAK2/STAT3 activation; thus suggesting that DEX treatment may be considered a potential strategy in the treatment of patients with SAP-ALI.

\section{Introduction}

Acute pancreatitis (AP) is an acute disease of the abdomen, which ranges from a mild, transient illness to a fatal disease with a mortality rate of $\sim 30 \%$. In addition, $25-30 \%$ of patients with severe AP (SAP) succumb to multiple organ system failure and pulmonary complications (1-4), which occur as a result of excessive inflammatory responses to pulmonary and extra-pulmonary stimuli, including pneumonia, acid aspiration, ischemia-reperfusion and sepsis (5). Resulting from pronounced systemic inflammatory response with increased endothelial and epithelial barrier permeability, gas exchange and oxygenation are compromised with leakage of a protein-rich exudate into the alveolar space and interstitial tissues (6). Excessive infiltration of polymorphonuclear leukocytes (PMNs) into the lungs has been identified as a key event in the development of acute lung injury (ALI) (7), and pulmonary microvascular endothelial cells (PMVECs) have been associated with the pathogenesis of ALI. Major events in the inflammatory response include the migration of leukocytes from the blood, their adhesion to the vascular endothelium, and their transmigration across the endothelium. Various types of adhesion molecules on the surface of leukocytes and endothelium have previously been associated with the cell adhesion process $(8,9)$. Intercellular cell adhesion molecule-1 (ICAM-1), which is a well-characterized adhesion molecule expressed in PMVECs, can be stimulated by proinflammatory cytokines, including tumor necrosis factor 
(TNF)- $\alpha$, interleukin (IL)-6 and interferon- $\gamma(10,11)$, which act via signaling pathways, including phosphoinositide 3-kinase, mitogen-activated protein kinases (MAPKs), and the nuclear factor $(\mathrm{NF})-\kappa \mathrm{B}$ pathway $(12,13)$.

The Janus kinase/signal transducer and activator of transcription (JAK/STAT) pathway (14) has been widely researched for its role in tumorigenesis. IL-6, which is a proinflammatory cytokine that preferentially activates STAT3, has been well recognized for its role in initiating and amplifying inflammatory processes. During inflammation, the adhesion molecules expressed on stimulated endothelial cells (ECs) are essential for the recruitment and transmigration of leukocytes into the subendothelial matrix (15). The induction of ICAM-1 has previously been associated with leukocyte adhesion and transmigration, resulting in damage to ECs and amplification of inflammatory responses. In addition, it has previously been reported that the effects of cholinergic agonists on IL-6-mediated ICAM-1 expression, and monocyte chemoattractant protein (MCP)-1 production by ECs, may be mediated via the JAK2/STAT3 signaling pathway (16). Dexamethasone, a synthetic adrenocortical steroid, is increasingly used in the treatment of SAP (17). Dexamethasone exerts anti-inflammatory, anti-toxicity and anti-inflammatory effects in patients with SAP, in addition to reducing the systemic inflammatory response to liver and lung damage.

In the present study, the expression levels of ICAM-1 in the pulmonary tissues of a rat model of SAP-ALI were investigated, in order to elucidate the underlying molecular mechanisms. ICAM-1 expression was shown to be induced by SAP via the JAK2/STAT3 signaling pathway, but could be suppressed by treatment with dexamethasone (DEX). In addition, DEX treatment was able to attenuate the inflammatory responses in order to protect the lungs against SAP-ALI.

\section{Materials and methods}

Rats. Male Sprague Dawley rats (body weight, 170-220 g) were purchased from the Specific Pathogen Free Animal Laboratory of Dalian Medical University (Dalian, China), and were housed in a controlled environment. Prior to the experiments, the rats were deprived of food; however, drinking water was available ad libitum. The rats were anesthetized using intraperitoneal administration of $10 \%$ chloral hydrate ( $3 \mathrm{ml} / \mathrm{kg}$; Baier Di Biotechnology Co., Ltd., Beijing, China), and the bile-pancreatic duct was exposed. A rat model of SAP was induced via a standardized pressure-controlled retrograde infusion of $1.5 \%$ deoxycholic acid sodium salt $(1 \mathrm{mg} / \mathrm{kg}$; Baier Di Biotechnology Co., Ltd.) into the bile-pancreatic duct, after which the bile-pancreatic duct near the margin of the liver and duodenum was compressed with a clip for $5 \mathrm{~min}$. Subsequently, the clip was removed and the abdomen was closed. All rats received humane care in compliance with the Public Health Service Policy on Humane Care, and use of the rats was approved by the Institutional Animal Ethics Committee of Dalian Medical University (Dalian, China).

Experimental groups. A total of 30 male Sprague Dawley rats were randomly divided into three groups, as follows ( $n=10 /$ group): i) Control (CON) group, in which the abdomens of the rats were opened, the pancreas was turned over and the abdomen was closed; ii) SAP group, in which SAP was induced via retrograde infusion of $1.5 \%$ deoxycholic acid sodium salts into the bile-pancreatic duct; and iii) DEX group, in which SAP was induced and the rats were subsequently treated with $1 \mathrm{ml} / \mathrm{kg}$ DEX (Henan Lingrui Pharmaceutical Co., Ltd., Zhengzhou, China), which was administered via the vena sublingualis, 5 min post-surgery. All rats were examined 24 h post-surgery. The rats were drained of blood and lung tissue samples were collected and stored at $-80^{\circ} \mathrm{C}$ for subsequent analysis.

Determination of ICAM-1, JAK2, STAT3 and NF- $\kappa B$ mRNA expression levels. Total cellular RNA was extracted from the cells 24 h post-surgery using RNAiso Plus (Takara Bio, Inc., Otsu, Japan) according to the manufacturer's protocol. The mRNA expression levels of ICAM-1, JAK2, STAT3 and $\mathrm{NF}-\kappa \mathrm{B}$ were quantified using reverse transcription-quantitative polymerase chain reaction (RT-qPCR), in which the Maxima SYBR-Green/ROX qPCR Master Mix (2X) (Thermo Fisher Scientific, Inc., Waltham, MA, USA) was used. RNA was solubilized in ribonuclease-free water and quantified by measuring the absorbance at $260 \mathrm{~nm}$ using an Ultrospec 2100 Pro spectrophotometer (GE Healthcare, Buckinghamshire, UK). The concentration was $220-280 \mathrm{ng} / \mu 1$ [the purity of RNA was confirmed by examining the optical density (OD) 260/280 as 1.6/1.9]. cDNA was synthesized from $1 \mu \mathrm{l}$ RNA using the Bio-Rad iScript ${ }^{\mathrm{TM}}$ cDNA Synthesis kit (Bio-Rad Laboratories, Inc. Hercules, CA, USA). Amplification and detection of $500 \mathrm{ng}$ cDNA was conducted using the Rotor Gene $3000^{\mathrm{TM}}$ sequence detection system (Qiagen, Inc., Valencia, CA, USA).

The primers and probes (Takara Bio, Dalian, China) used were as follows: ICAM-1 forward, 5'-GCTTCTGCCACCATC ACTGTGTA-3' and reverse, 5'-ATGAGGTTCTTGCCCACC TG-3'; JAK2 forward, 5'-TTTGAAGACAGGGACCCTACA CAG-3' and reverse, 5'-TCATAGCGGCACATCTCCACA-3'; STAT3 forward, 5'-CACCCATAGTGAGCCCTTGGA-3' and reverse, 5'-TGAGTGCAGTGACCAGGACAGA-3'; NF-кB forward, 5'-GATGGGACGACACCTCTACACATA-3' and reverse, 5'-CCCAAGAGTCGTCCAGGTCA-3'; and $\beta$-actin forward, 5'-GGAGATTACTGCCCTGGCTCCTA-3' and reverse, 5'-GACTCATCGTACTCCTGCTTGCTG-3'. $\beta$-actin was used as an internal control. The PCR cycling conditions included 40 cycles of $95^{\circ} \mathrm{C}$ for $5 \mathrm{sec}$ and $60^{\circ} \mathrm{C}$ for $31 \mathrm{sec}$, as per manufacturer's protocol. This was followed by an annealing step at $95^{\circ} \mathrm{C}$ for $15 \mathrm{sec}$ followed by a cycle at $60^{\circ} \mathrm{C}$ for $60 \mathrm{sec}$ and a final step at $95^{\circ} \mathrm{C}$ for $15 \mathrm{sec}$. For relative quantification, the copy ratios of ICAM- $1 / \beta$-actin, JAK $2 / \beta$-actin, STAT3 $/ \beta$-actin and $\mathrm{NF}-\kappa \mathrm{B} / \beta$-actin, were calculated and used as an indication of the relative expression levels.

Western blot analysis. Frozen lung tissue was mechanically homogenized in $1 \mathrm{ml}$ ice-cold extraction buffer, containing $50 \mathrm{mmol} / 1$ Tris-His (pH 7.4), 1\% NP-40.0, 25\% sodium deoxycholate, $150 \mathrm{mmol} / 1 \mathrm{NaCl}, 1 \mathrm{mmol} / 1$ ethylene diamine tetraacetic acid, $1 \mathrm{mmol} / 1$ phenylmethylsulfonyl fluoride, $0.1 \%$ sodium dodecylsulfate, and $1 \mu \mathrm{g} / \mathrm{ml}$ each of aprotinin and leupeptin. Following incubation on ice for $30 \mathrm{~min}$, the homogenate was centrifuged at $12,000 \times \mathrm{g}$ for $10 \mathrm{~min}$ at $4^{\circ} \mathrm{C}$, after which the supernatant was stored at $-80^{\circ} \mathrm{C}$ prior to analysis. The protein concentration was determined using the Bradford Protein Assay (Thermo Fisher Scientific, Inc.), with bovine serum 
albumin as a standard. Equal volumes of each sample $(10 \mu \mathrm{g})$ were separated by $12 \%$ sodium dodecyl sulfate-polyacrylamide gel electrophoresis, and transferred to a polyvinylidene difluoride membrane (Nanjing KeyGen Biotech Co., Ltd., Nanjing, China). Subsequently, the membrane was incubated with $200 \mu \mathrm{g} / \mathrm{ml}$ goat polyclonal anti-rat ICAM-1 antibodies (1:500; sc-1511; Santa Cruz Biotechnology, Inc., Dallas, TX, USA) overnight at $4^{\circ} \mathrm{C}$, prior to washing with Tris-buffered saline (TBS) containing $0.05 \%$ Tween-20. The membrane was then incubated with horseradish peroxidase (HRP)-conjugated goat anti-rat ( $500 \mu \mathrm{g} / \mathrm{ml} ; 1: 10,000 ;$ ab6721; Abcam, Cambridge, UK) for $1 \mathrm{~h}$ at room temperature. The antibody-antigen complexes were detected using an enhanced chemiluminescence reagent (Immobilon Western HRP Substrate; WBKLS0100; EMD Millipore, Billerica, MA, USA), and exposed to X-OMAT BT Film (Eastman Kodak, Rochester, NY, USA). Films were scanned on a UniScan C800 (Tsinghua UniSplendour Co., Ltd., Beijing, China) using Photoshop software (Adobe Systems, Inc., San Jose, CA, USA), and the OD of each band was determined using Gel-Pro Analyzer 4.0 software (Media Cybernetics, Inc., Rockville, MD, USA).

Enzyme-linked immunosorbent assay (ELISA). An ELISA was performed in order to quantify the concentrations of IL-6, IL-8 and TNF- $\alpha$ in the serum samples. Commercially available kits were used (R\&D Systems, Inc., Minneapolis, MN, USA), following the manufacturer's instructions.

Immunohistochemical staining and immunofluorescence. The rat lungs were infused with $10 \%$ buffered formalin for $24 \mathrm{~h}$ and embedded in paraffin. Subsequently, $5 \mu \mathrm{m}$ tissue sections were prepared, and continuous sections of the paraffin-embedded tissue were taken for pathological examination using hematoxylin-eosin staining (G1120-100; Beijing Solarbio Science \& Technology Co., Ltd. Beijing, China). After deparaffinization, the tissue sections were treated with sodium citrate and boiled for $2 \mathrm{~min}$ in a pressure cooker for restoration. After washing three times with phosphate-buffered saline (PBS), the tissue sections were incubated for $10 \mathrm{~min}$ with $3 \%$ hydrogen peroxide at room temperature. Subsequently, the lung tissue sections were incubated for $1 \mathrm{~h}$ with rabbit anti-rat JAK2 (1:50; sc-294) and STAT3 (1:50; sc-482) antibodies (Santa Cruz Biotechnology, Inc.) at room temperature. After washing with PBS, the tissue sections were incubated for $30 \mathrm{~min}$ at room temperature with poly-peroxidase-conjugated anti-rabbit immunoglobulin G. Diaminobenzidine (Baier Di Biotechnology Co., Ltd.) was used as a substrate for the immunoperoxidase reaction. After rinsing with water, the tissue sections were stained with hematoxylin and incubated with goat anti-rat ICAM-1 and NF- $\mathrm{KB}$ antibodies for $1 \mathrm{~h}$. Imunofluorescence was detected following incubation of the tissue sections with 4',6-diamidino-2-phenylindole for $10 \mathrm{~min}$. The tissue samples were observed using an inverted fluorescence microscope (Leica Microsystems, Mannheim, Germany). Imaging was conducted using the Image-Pro Plus 6.0 software (Media Cybernetics, Inc.).

Statistical analysis. The target protein and $\beta$-actin band intensities were analyzed using Image J software, version $1.35 \mathrm{~d}$ (National Institutes of Health, Bethesda, MD, USA). The target protein $/ \beta$-actin groups were then compared with the

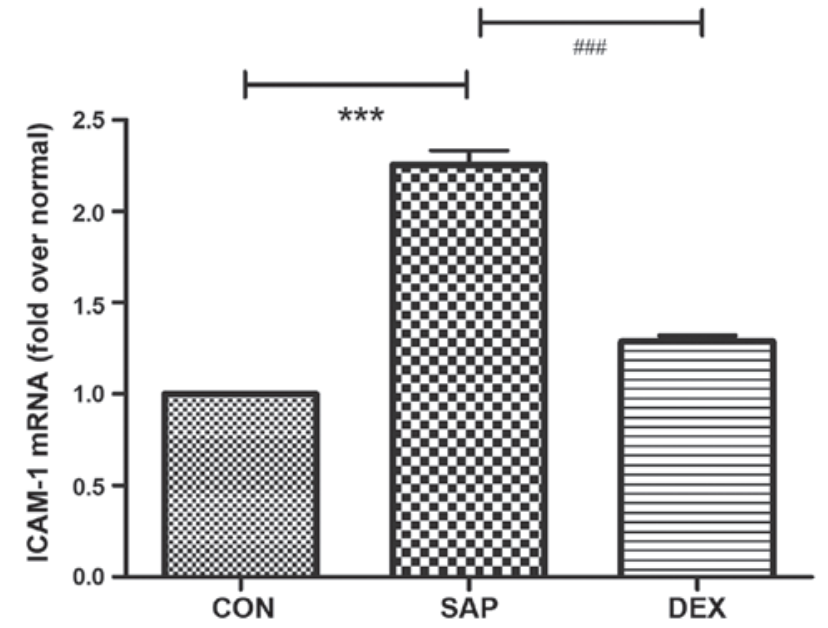

Figure 1. mRNA expression levels of ICAM-1 were determined using reverse transcription-quantitative polymerase chain reaction. ICAM-1 mRNA expression levels were significantly increased in the SAP group, as compared with the CON group. ICAM-1 mRNA expression levels were significantly decreased in the DEX-treated group, as compared with the SAP group. Data are presented as the mean \pm standard deviation. ${ }^{* * *} \mathrm{P}<0.01$ vs. the CON group;

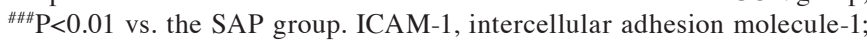
SAP, severe acute pancreatitis; CON, control; DEX, dexamethasone.

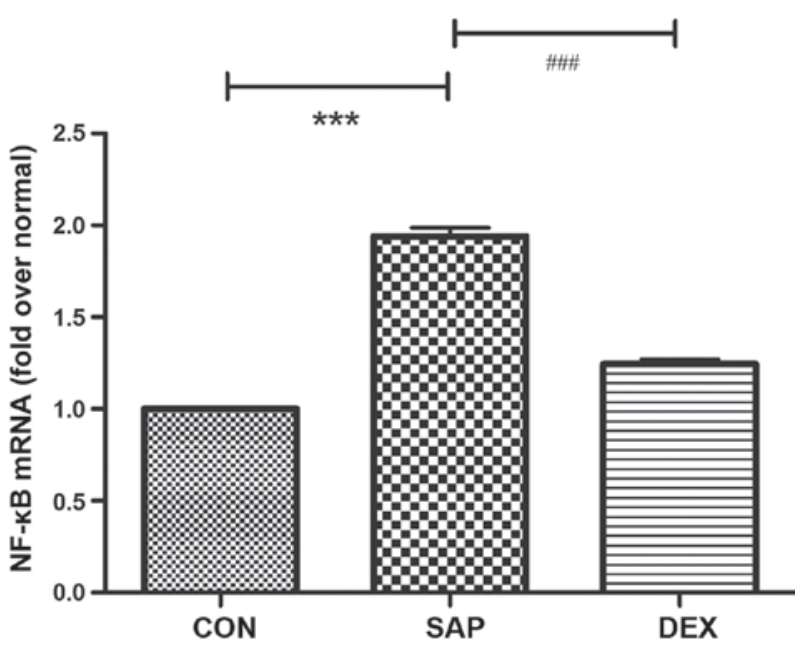

Figure 2. mRNA expression levels of NF- $\kappa \mathrm{B}$ were determined using reverse transcription-quantitative polymerase chain reaction. NF- $\kappa \mathrm{B}$ mRNA expression levels were significantly increased in the SAP group, as compared with the CON group. NF- $\kappa \mathrm{B}$ mRNA expression levels were significantly decreased in the DEX-treated group, as compared with the SAP group. Data are presented as the mean \pm standard deviation. ${ }^{* * * *} \mathrm{P}<0.01$ vs. the CON group;

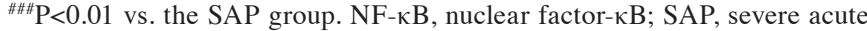
pancreatitis; CON, control; DEX, dexamethasone.

control/ $\beta$-actin group, statistically analyzed with SPSS software, version 16.0 (SPSS, Inc., Chicago, IL, USA). Data are presented as the mean \pm standard deviation. Groups were compared using one-way analysis of variance and the Student Newman-Keuls method. $\mathrm{P}<0.05$ was considered to indicate a statistically significant difference.

\section{Results}

ICAM-1 and NF- $\kappa B$ expression levels are increased following SAP induction and decreased following DEX treatment. In 

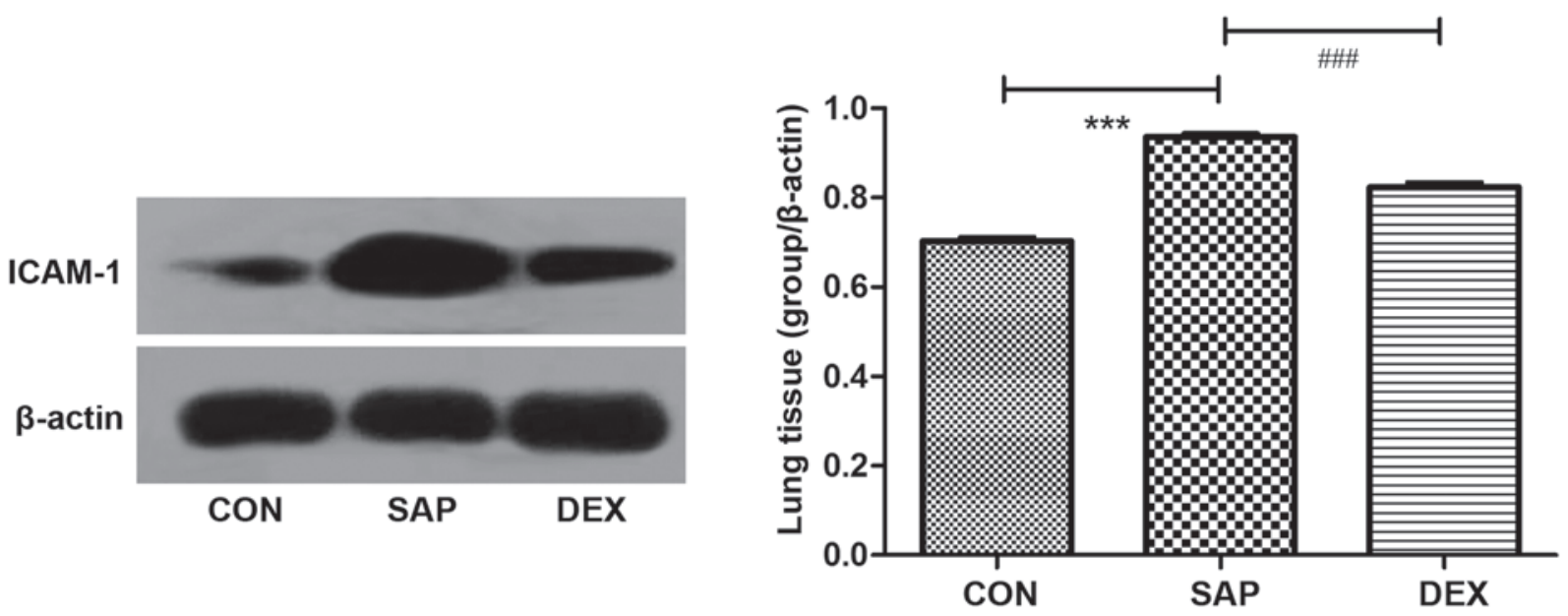

Figure 3. ICAM-1 protein expression levels were significantly increased in the SAP group, as compared with the CON group, as demonstrated by western blotting. ICAM-1 protein expression levels in the DEX-treated group were significantly decreased, as compared with the SAP group. $\beta$-actin was used as an internal control. Data are presented as the mean \pm standard deviation. ${ }^{* * *} \mathrm{P}<0.01$ vs. the CON group; ${ }^{\# \# ~} \mathrm{P}<0.01$ vs. the SAP group. ICAM-1, intercellular adhesion molecule-1; SAP, severe acute pancreatitis; CON, control; DEX, dexamethasone.

A

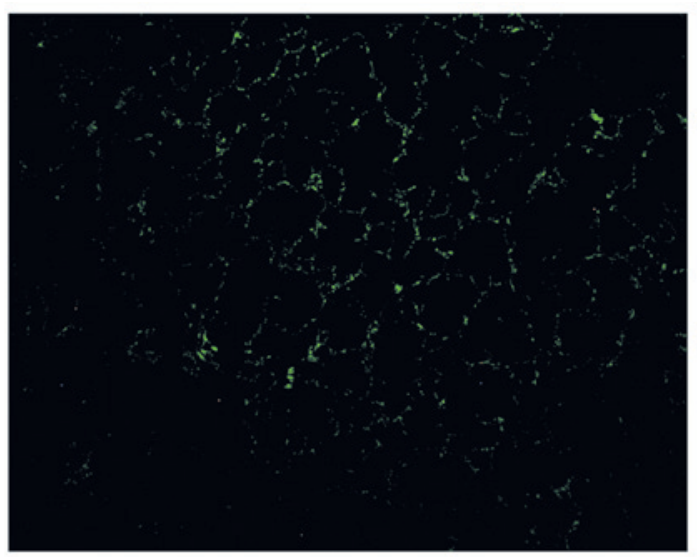

C

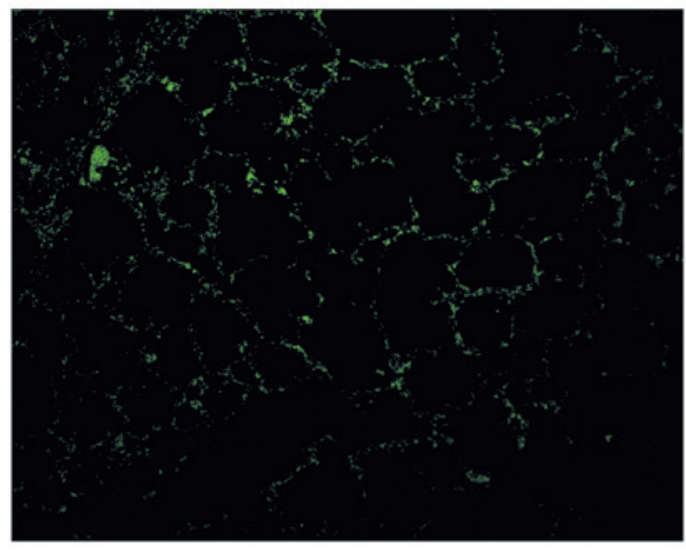

B


Figure 4. Representative ICAM-1 immunofluorescence of the lung tissues from the (A) CON, (B) SAP, and (C) DEX-treated groups at $24 \mathrm{~h}$ following induction of SAP (magnification, 40x). (D) Comparison of the IOD values for ICAM-1 in lung tissues. Data are presented as the mean \pm standard deviation. ${ }^{* * *} \mathrm{P}<0.01 \mathrm{vs}$. the CON group; ${ }^{\# \# "} \mathrm{P}<0.01$ vs. the SAP group. ICAM-1, intercellular adhesion molecule-1; SAP, severe acute pancreatitis; CON, control; DEX, dexamethasone; IOD, integrated optical density.

order to assess the role of ICAM-1 in SAP-ALI, the effects of SAP induction followed by DEX treatment on the mRNA and protein expression levels of ICAM-1 and NF- $\kappa \mathrm{B}$ were investigated in a rat model of SAP-ALI. ICAM-1 and NF- $\kappa$ B mRNA expression levels in the lung tissue sections from the CON group at $24 \mathrm{~h}$ following SAP injury were used as a baseline. Similar alterations in mRNA expression levels were detected for ICAM-1 and NF-кB in the SAP group (Figs. 1 and 2), and 
A

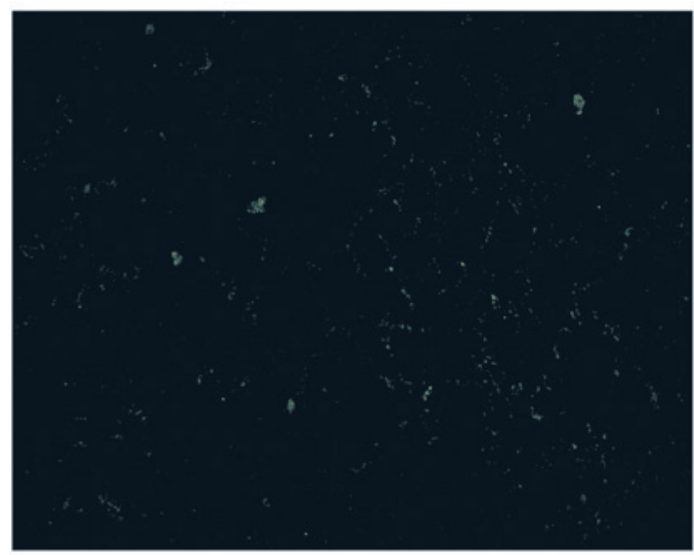

C

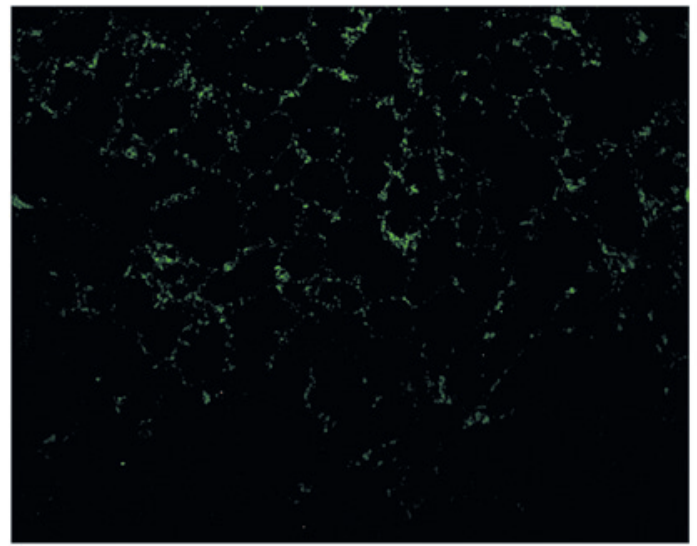

B

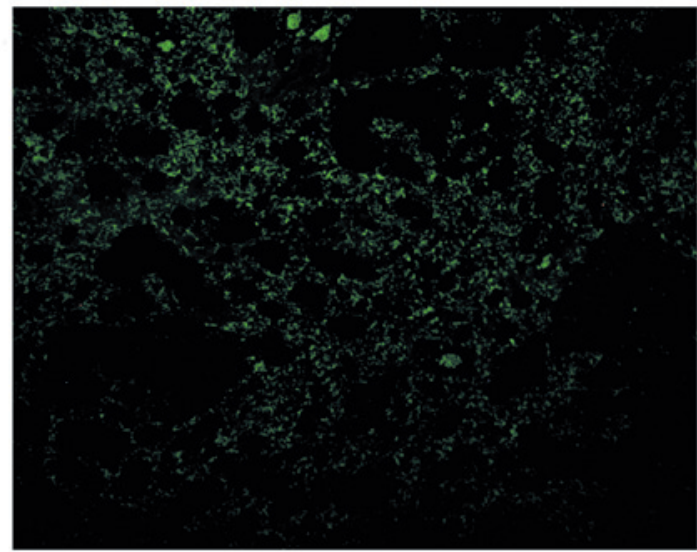

D

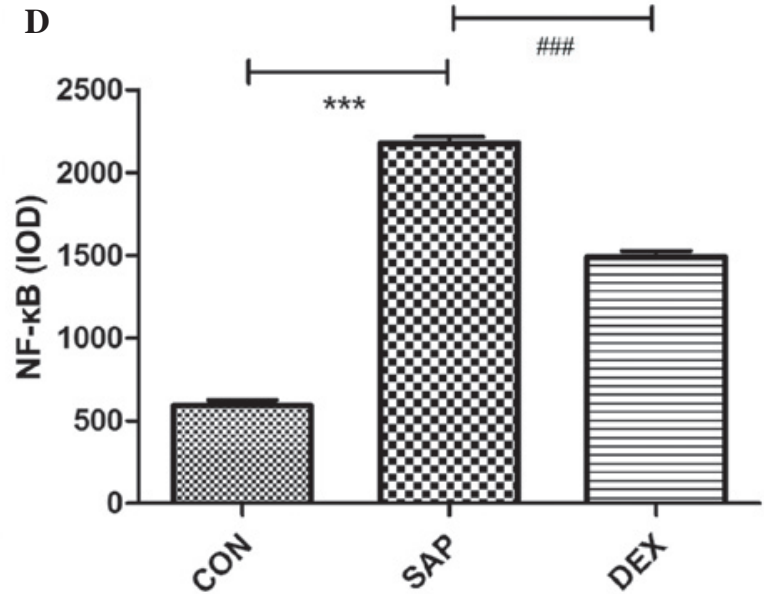

Figure 5. Representative NF- $\kappa$ B immunofluorescence of the lung tissue sections from the (A) CON, (B) SAP and (C) DEX-treated groups at 24 h following induction of SAP (magnification, 40x). (D) Comparison of the IOD values for ICAM-1 in lung tissue sections: ${ }^{* * *} \mathrm{P}<0.01$ vs. the CON group; ${ }^{\# \#} \mathrm{P}<0.01$ vs. the SAP group. NF- $\kappa \mathrm{B}$, nuclear factor- $\kappa \mathrm{B}$; SAP, severe acute pancreatitis; CON, control; DEX, dexamethasone; IOD, integrated optical density.

were characterized by a significant increase following SAP induction $(\mathrm{P}<0.01$; Figs. 1 and 2$)$. DEX treatment significantly decreased the mRNA expression levels of ICAM-1 and NF- $\mathrm{BB}$ in the lung tissue post-injury, as compared with those in the SAP group $(\mathrm{P}<0.01$; Figs. 1 and 2$)$.

The protein expression levels of ICAM-1 in the lung tissue of the rats were analyzed by western blotting and immunofluorescence. Western blotting demonstrated that ICAM-1 protein expression levels were significantly increased in the SAP group, as compared with the CON group $(\mathrm{P}<0.01$; Fig. 3$)$. Conversely, ICAM-1 protein expression levels were significantly decreased in the DEX-treated group, as compared with the SAP group ( $\mathrm{P}<0.01$; Fig. 3). Low levels of ICAM-1 were detected on the vascular endothelial and bronchiolar and alveolar epithelial surfaces of the CON group using immunofluorescence (Fig. 4A), and these were significantly increased in the SAP group at $24 \mathrm{~h}$ following SAP induction $(\mathrm{P}<0.01$; Fig. 4B). Conversely, in the rats treated with DEX, ICAM-1 levels were significantly decreased, as compared with those in the SAP group $(\mathrm{P}<0.01$; Fig. $4 \mathrm{C})$. These results suggest that DEX is able to decrease the SAP-induced expression of ICAM-1 (Fig. 4D).

The protein expression levels of $\mathrm{NF}-\kappa \mathrm{B}$ were analyzed using immunofluorescence, which demonstrated that the levels of $\mathrm{NF}-\kappa \mathrm{B}$ were significantly increased in the SAP group, as compared with the CON group $(\mathrm{P}<0.01$; Fig. 5). Conversely,
$\mathrm{NF}-\kappa \mathrm{B}$ protein expression levels were significantly decreased in the DEX-treated group, as compared with the SAP group $(\mathrm{P}<0.01$; Fig. 5). These results suggest that DEX is able to decrease the SAP-induced upregulation of NF- $\kappa \mathrm{B}$.

IL-6, IL-8 and TNF- $\alpha$ concentrations increase following SAP induction. The concentrations of IL- 6 , IL- 8 and TNF- $\alpha$ in plasma were detected using an ELISA. In the CON group, the concentrations of IL-6, IL- 8 and TNF- $\alpha$ were 216.06 \pm 11.04 , $119.28 \pm 15.2$ and $225.97 \pm 10.56 \mathrm{ng} / 1$, respectively. The concentrations of the cytokines were significantly increased following SAP induction to $310.47 \pm 12.28,270.06 \pm 38.36$ and $309.16 \pm 10.62 \mathrm{ng} / 1$, respectively $(\mathrm{P}<0.01$; Fig. 6). Treatment with DEX significantly inhibited the SAP-induced production of IL-6, IL-8 and TNF- $\alpha(\mathrm{P}<0.01$; Fig. 6).

Analysis of the JAK2/STAT3 signaling pathway in lung tissue. A previous study demonstrated that IL-6-induced MCP-1 production by HUVECs was mediated by the JAK2/STAT3 pathway (15). In order to evaluate the role of this signaling cascade in SAP-ALI-induced ICAM-1 expression, the protein expression levels of JAK2 and STAT3 were analyzed by immunohistochemistry. The protein expression levels of JAK2 and STAT3 in the lung tissue sections were significantly upregulated in the SAP group, as compared with the CON group $(\mathrm{P}<0.01$; Figs. 7 and 8$)$. Conversely, JAK2 and STAT3 

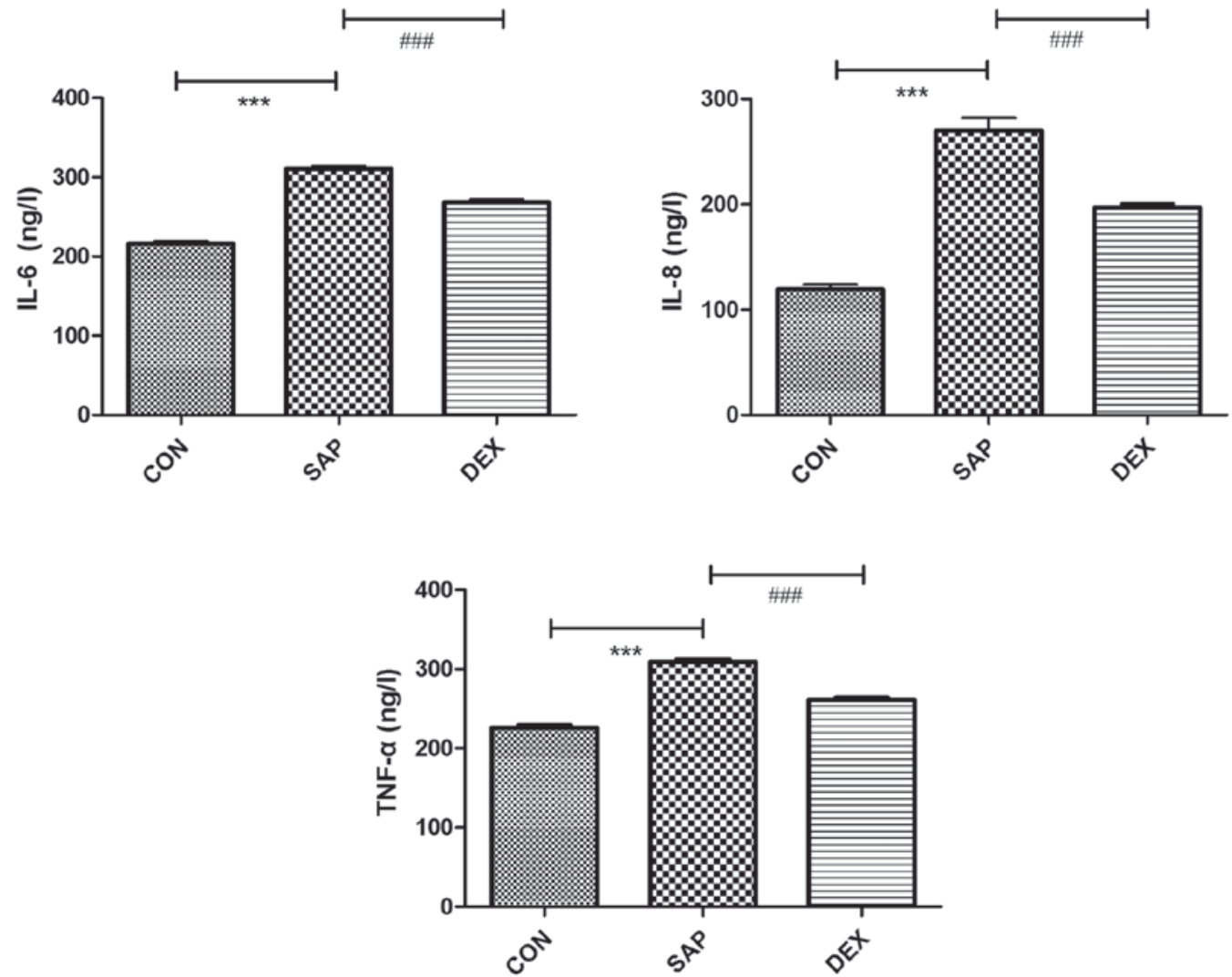

Figure 6. Serum IL-6, IL-8 and TNF- $\alpha$ levels were determined using an ELISA. Data are presented as the mean \pm standard deviation. ${ }^{* * * *} \mathrm{P}<0.01 \mathrm{vs}$. the CON group; ${ }^{\# \# ~} \mathrm{P}<0.01$ vs. the SAP group. IL, interleukin; TNF, tumor necrosis factor; ELISA, enzyme-linked immunosorbent assay, SAP, severe acute pancreatitis; CON, control; DEX, dexamethasone.
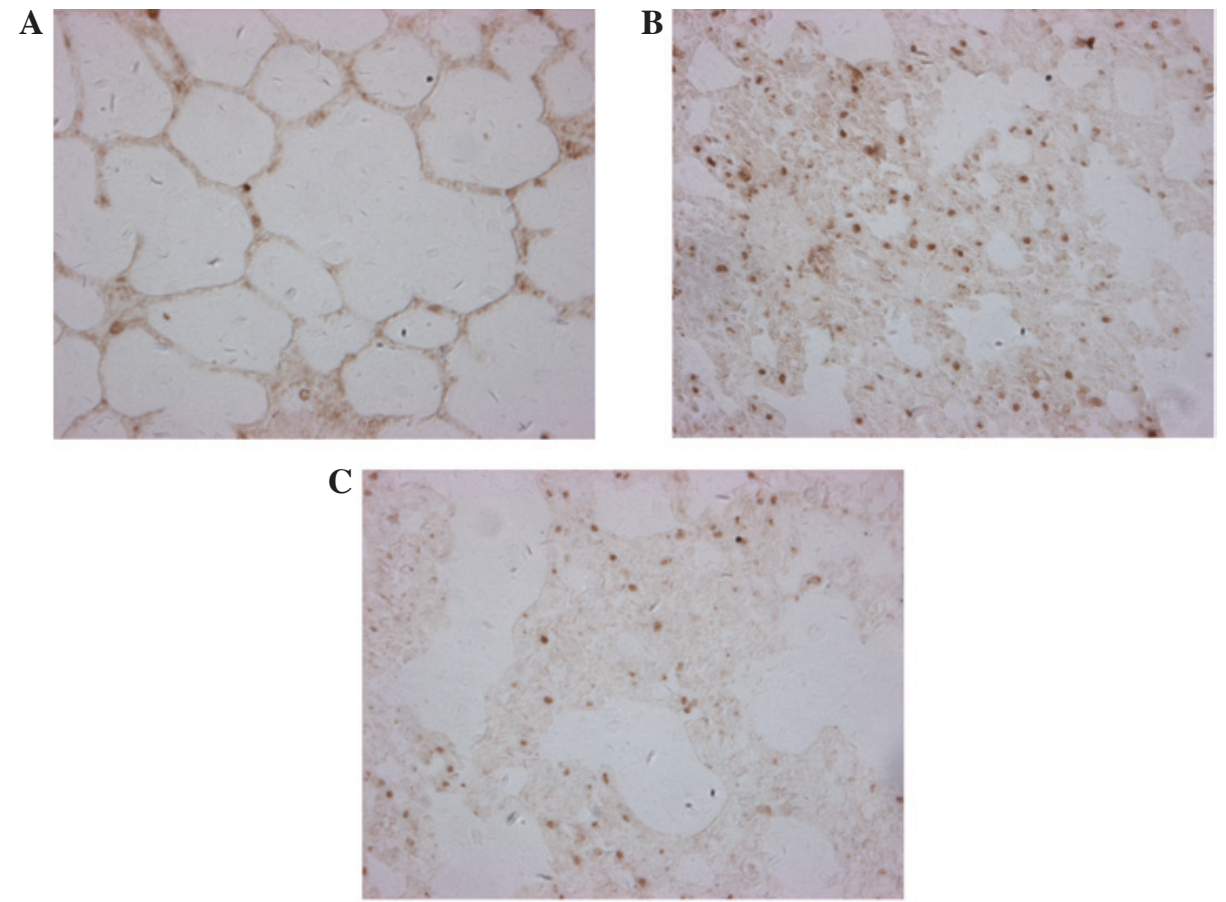

Figure 7. Representative immunohistochemical results for JAK2 in the lung tissue sections from the (A) CON, (B) SAP, and (C) DEX-treated groups at $24 \mathrm{~h}$ following induction of SAP (magnification, x40). JAK2, janus kinase 2; SAP, severe acute pancreatitis; CON, control; DEX, dexamethasone.

protein expression levels were significantly decreased in the DEX group, as compared with the SAP group (P<0.01; Figs. 7 and 8). These results suggest that the JAK2/STAT3 signaling pathway may upregulate ICAM-1 expression in response to SAP injury, and that DEX treatment is able to attenuate JAK2/STAT3 signaling. 

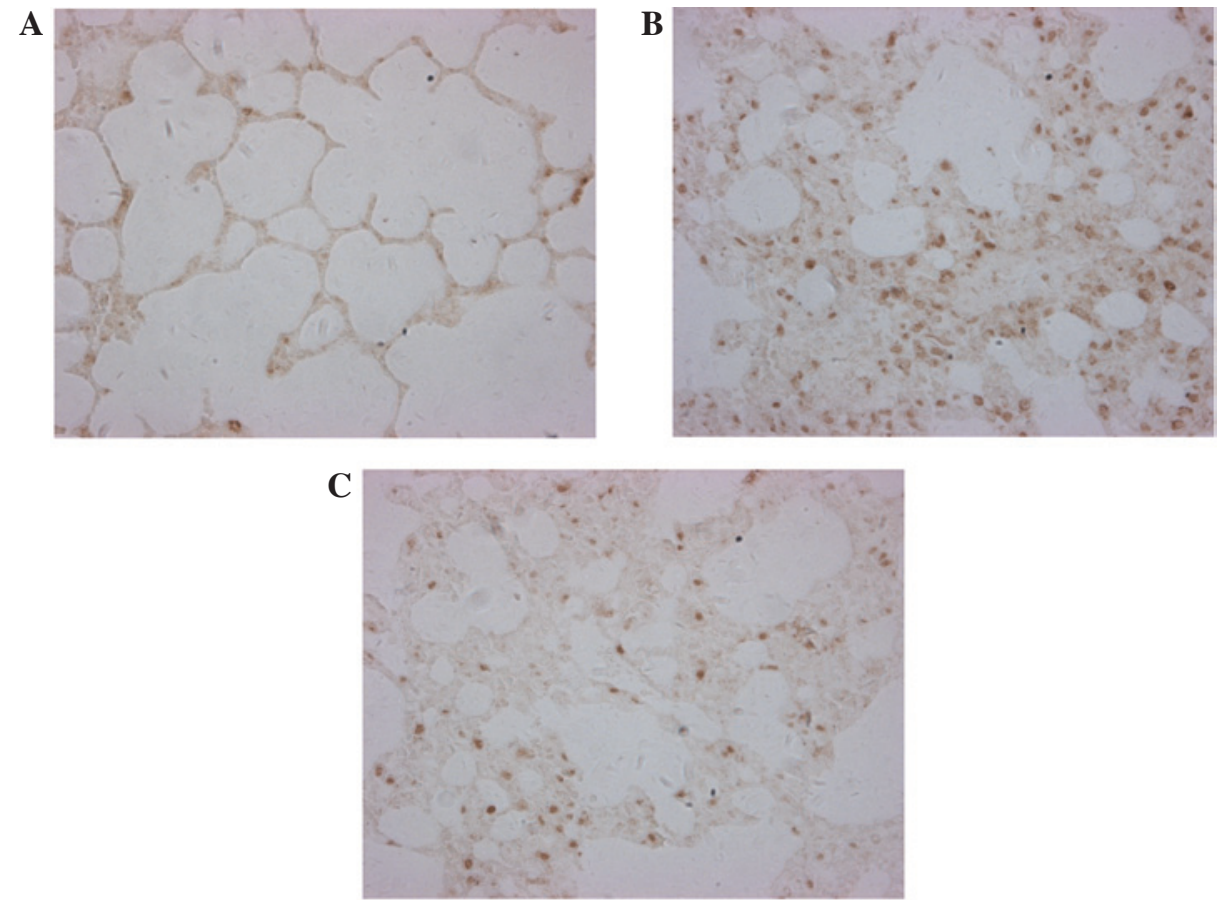

Figure 8. Representative immunohistochemical results for STAT3 of the lung tissue sections from the (A) CON, (B) SAP, and (C) DEX-treated groups at 24 $\mathrm{h}$ following induction of SAP (magnification, $\mathrm{x} 40$ ). STAT3, signal transducer and activator of transcription-3; SAP, severe acute pancreatitis; CON, control; DEX, dexamethasone.

Analysis of lung tissue morphology. ICAM-1 staining was increased in the lung tissue of the SAP group, as compared with the CON group (Fig. 4). The rats in the SAP group exhibited expanded and congested minute pulmonary vessels, and alveolar septum capillaries. In addition, the alveolar walls had burst, the alveolar space was narrowed, partial alveoli were damaged and the alveolar septum was broadened. Furthermore, there was marked infiltration of PMN cells into the alveoli and pulmonary interstitial tissue of the SAP rats, and this was associated with hemorrhage and pulmonary interstitial edema. Therefore, increased ICAM-1 staining was an indication of a severe injury. DEX treatment markedly reduced the severity of the SAP-induced pulmonary histopathological injury, as demonstrated by decreased protein expression levels of JAK2 and STAT3 in the DEX-treated rats, as compared with the SAP group. However, the role of the JAK2/STAT3 signaling pathway in SAP-induced upregulation of ICAM-1, IL6 and TNF- $\alpha$, should be investigated in future studies.

\section{Discussion}

Infiltration of monocytes and macrophages into local areas of inflammation in pulmonary tissues is a key event in SAP-ALI, and ICAM-1 has previously been shown to have an important role in mediating the inflammatory response (18). A previous study reported protective effects for angiopoietin-like protein-4 (Angpt14) in PMVECs in a rat model of acute inflammatory stroke (19). The present study demonstrated that the mRNA and protein expression levels of ICAM-1 were upregulated in the lung tissue of a rat model of SAP-ALI, and that this could be attenuated by treatment with DEX.

ICAM-1 (CD54) is an inducible surface glycoprotein with a molecular weight of $80-114 \mathrm{kDa}$, and is a member of the Ig superfamily, which consists of five Ig-like domains, a hydrophobic transmembrane domain and a short cytoplasmic C-terminal domain (20). Under stable conditions, ICAM-1 is expressed at low levels in endothelial and epithelial cells, or constitutively on the surface of alveolar cells, where it is involved in cell recognition, activation, proliferation, differentiation and motility, thereby helping to stabilize the internal environment of the body (10). During inflammation, ICAM-1 binds to two integrins, CD11a/CD18 (LFA-1) and CD11b/CD18 (Mac-1), which are members of the $\beta 2$ subfamily. LFA- 1 and Mac-1 are expressed by leukocytes and promote the adhesion and transendothelial migration of these cells $(20,21)$. In addition, ICAM-1 has a key role in pathological events associated with inflammatory reactions, including acute renal failure and acute pancreatitis (22).

Upregulation of ICAM-1 expression in the lungs during ALI, which is an often fatal complication of SAP, has previously been associated with leukocyte adhesion and activation, as well as induction of the 'cascade effect' of inflammatory mediators, pulmonary microcirculation dysfunction, acute respiratory distress syndrome (ARDS), multiple organ failure, and mortality (7,23). TNF- $\alpha$ has previously been shown to activate ICAM-1 expression via a $N F-\kappa B$ cis-element located in the 5'-flanking region of the ICAM-1 gene $(24,25)$. NF- $\kappa \mathrm{B}$ is a proinflammatory transcription factor that triggers inflammatory cascades during inflammatory responses, and $\mathrm{NF}-\kappa \mathrm{B}$ activation has been shown to regulate the expression of numerous genes encoding proinflammatory cytokines, chemokines, adhesion molecules and inducible enzymes $(26,27)$. The results of the present study suggested that high levels of TNF- $\alpha$ and NF- $\kappa \mathrm{B}$ may contribute to the development of SAP-ALI via induction of ICAM-1 expression. 
ECs under inflammatory stimulation produce cytokines, chemokines and cell adhesion molecules, which participate in various biological processes, including vascular remodeling and atherosclerosis (28); and therefore suppressing the release of these mediators is important for controlling inflammation. The present study demonstrated that the SAP-induced upregulation of the inflammation-associated proteins ICAM-1 and $\mathrm{NF}-\kappa \mathrm{B}$ in the lung tissue could be suppressed by DEX treatment, which is a widely used steroid that has previously been shown to exert anti-inflammatory and immunosuppressant effects (29). Serum ICAM-1 is an early diagnostic and predictive marker of SAP (30). Therefore, ICAM-1 may be considered a key target for potential attenuation of inflammation in patients with SAP-ALI.

The mechanism underlying SAP-induced ALI is currently unknown. Previous studies investigating MAPK signaling pathways have greatly enriched the current understanding of the molecular mechanisms underlying various biological stimuli, including inflammatory mediators (31-33). Wang et al (19) demonstrated that, in rat PMVECs treated with lipopolysaccharides, overexpression of Angptl4 or rosiglitazone treatment inhibited the Raf/[MAPK/extracellular signal-regulated kinase (ERK)]/MAPK cascade and was able to protect against increased permeability induced by F-actin depolymerization. In addition, phosphorylated-AKT/AKT downregulation was shown to inactivate phosphorylation of $\mathrm{NF}-\kappa \mathrm{B}$, inhibiting the downstream production of inflammatory cytokines, including TNF- $\alpha$ (19,34). Conversely, the present study demonstrated that JAK2/STAT3 levels were upregulated in a SAP-ALI rat model, and were decreased following treatment with DEX. Previous studies have demonstrated a role for DEX in reducing STAT3 expression levels (35). In the present study, JAK2 and STAT3 upregulation was associated with inflammation in a rat model of SAP-ALI, and may have also been involved in cellular responses to various cytokines, growth factors and hormones. Further experiments are required in order to explore the network of MAPK/ERK/JAK signaling that may contribute to the SAP-ALI-associated inflammatory response.

ALI/ARDS is an inflammatory injury of the lungs that is predominantly characterized by PMN cell infiltration (36). It has previously been reported that the flavonols, luteolin, apigenin and quercetin, were able to inhibit ICAM-1 mRNA and protein expression by inactivating $N F-\kappa B$ in TNF- $\alpha$-stimulated HUVECs (37). Furthermore, it has been demonstrated that 2'-hydroxychalcone, an achalcone derivative, was able to attenuate TNF- $\alpha$-induced ICAM-1 expression via NF- $\kappa \mathrm{B}$ inactivation, leading to reduced adhesion of neutrophils to HUVECs (38). In the present study, SAP-induced TNF- $\alpha$ and $N F-\kappa B$ upregulation was shown to be attenuated by DEX treatment; however, a previous study suggested that DEX treatment was unable to entirely attenuate leukocyte recruitment during sodium taurocholate-induced acute pancreatitis (39). DEX treatment may attenuate cytokine production via inhibition of ICAM-1; a possible explanation for its anti-inflammatory effect. However, this does not fully explain the ability of DEX to inhibit the production of cytokines and inflammatory factors; thus suggesting that further research is required.

In conclusion, the present study demonstrated that in vivo activation of JAK2/STAT3 signaling may be involved in the pathogenesis of SAP-ALI. These results suggested that ICAM-1 and JAK/STAT signaling intermediates, as well as components of other acute inflammatory signaling pathways, including the MAPK pathway, may be important targets for intervention in the treatment of patients with SAP-ALI. The present study demonstrated that downregulation of the ICAM-1-mediated JAK2/STAT3 signaling cascade was able to attenuate inflammatory responses that promote leukocyte trafficking during inflammation. Further elucidation of the molecular mechanisms underlying SAP-ALI will be crucial for the development of novel treatments in the future.

\section{Acknowledgements}

The present study was supported by The National Natural Science Foundation of China (grant no. 81173452). The present study was conducted at the Laboratory of the First Affiliated Hospital of Dalian Medical University and Dalian Center Hospital (Dalian, China). The authors would like to thank Dr Hao Zhang for assistance with editing.

\section{References}

1. Chen C, Xu S, Wang WX, Ding YM, Yu KH, Wang B and Chen XY: Rosiglitazone attenuates the severity of sodium taurocholate-induced acute pancreatitis and pancreatitis-associated lung injury. Arch Med Res 40: 79-88, 2009.

2. Lund H, Tønnesen H, Tønnesen MH and Olsen O: Long-term recurrence and death rates after acute pancreatitis. Scand J Gastroenterol 41: 234-238, 2006.

3. Renzulli P, Jakob SM, Täuber M, Candinas D and Gloor B: Severe acute pancreatitis: Case-oriented discussion of interdisciplinary management. Pancreatology 5: 145-156, 2005.

4. Williams M and Simms HH: Prognostic usefulness of scoring systems in critically ill patients with severe acute pancreatitis. Crit Care Med 27: 901-907, 1999.

5. Storme L, Aubry E, Rakza T, Houeijeh A, Debarge V, Tourneux P, Deruelle P and Pennaforte T; French Congenital Diaphragmatic Hernia Study Group: Pathophysiology of persistent pulmonary hypertension of the newborn: Impact of the perinatal environment. Arch Cardiovasc Dis 106: 169-177, 2013.

6. Shields CJ, Winter DC and Redmond HP: Lung injury in acute pancreatitis: Mechanisms, prevention and therapy. Curr Opin Crit Care 8: 158-163, 2002.

7. Yuan Q, Jiang YW, Ma TT, Fang QH and Pan L: Attenuating effect of Ginsenoside Rb1 on LPS-induced lung injury in rats. J Inflamm (Lond) 11: 40, 2014.

8. Rao RM, Yang L, Garcia-Cardena G, and Luscinskas FW: Endothelial-dependent mechanisms of leukocyte recruitment to the vascular wall. Circ Res 101: 234-247, 2007.

9. Williams MR, Azcutia V, Newton G, Alcaide P and Luscinskas FW: Emerging mechanisms of neutrophil recruitment across endothelium. Trends Immunol 32: 461-469, 2011.

10. Meager A: Cytokine regulation of cellular adhesion molecule expression in inflammation. Cytokine Growth Factor Rev 10: 27-39, 1999.

11. Roebuck KA and Finnegan A: Regulation of intercellular adhesion molecule-1 (CD54) gene expression. J Leukoc Biol 66: 876-888, 1999.

12. Minhajuddin M, Bijli KM, Fazal F, Sassano A, Nakayama KI, Hay N, Platanias LC and Rahman A: Protein kinase C-delta and phosphatidylinositol 3-kinase/Akt activate mammalian target of rapamycin to modulate NF-kappaB activation and intercellular adhesion molecule-1 (ICAM-1) expression in endothelial cells. J Biol Chem 284: 4052-4061, 2009.

13. Amin MA, Haas CS,Zhu K, Mansfield PJ, Kim MJ,Lackowski NP and Koch AE: Migration inhibitory factor up-regulates vascular cell adhesion molecule-1 and intercellular adhesion molecule-1 via Src, PI3 kinase, and NFkappaB. Blood 107: 2252-2261, 2006.

14. Boengler K, Hilfiker-Kleiner D, Drexler H, Heusch G and Schulz R: The myocardial JAK/STAT pathway: From protection to failure. Pharmacol Ther 120: 172-185, 2008. 
15. Chen SC, Chang YL, Wang DL and Cheng JJ: Herbal remedy magnolol suppresses IL-6-induced STAT3 activation and gene expression in endothelial cells. Br J Pharmacol 148: 226-232, 2006.

16. Chatterjee PK, Al-Abed Y, Sherry B and Metz CN: Cholinergic agonists regulate JAK2/STAT3 signaling to suppress endothelia cell activation. Am J Physiol Cell Physiol 297: C1294-C1306, 2009.

17. Dong LH, Liu ZM, Wang SJ, Zhao SJ, Zhang D, Chen Y and Wang YS: Corticosteroid therapy for severe acute pancreatitis: A meta-analysis of randomized, controlled trials. Int J Clin Exp Pathol 8: 7654-7660, 2015.

18. Kojima R, Kawachi M and Ito M: Butein suppresses ICAM-1 expression through the inhibition of IкB $\alpha$ and c-Jun phosphorylation in TNF- $\alpha$ - and PMA-treated HUVECs. Int Immunopharmacol 24: 267-275, 2015.

19. Wang Y, Chen H, Li H, Zhang J and Gao Y: Effect of angiopoietin-like protein 4 on rat pulmonary microvascular endothelial cells exposed to LPS. Int J Mol Med 32: 568-576, 2013.

20. Staunton DE, Marlin SD, Stratowa C, Dustin ML and Springer TA: Primary structure of ICAM-1 demonstrates interaction between members of the immunoglobulin and integrin supergene families. Cell 52: 925-933, 1988.

21. Diamond MS, Staunton DE, Marlin SD and Springer TA Binding of the integrin Mac-1 (CD11b/CD18) to the third immunoglobulin-like domain of ICAM-1 (CD54) and its regulation by glycosylation. Cell 65: 961-971, 1991.

22. Yang N, Luo M, Li R, Huang Y, Zhang R, Wu Q, Wang F, Li Y and Yu X: Blockage of JAK/STAT signalling attenuates renal ischaemia-reperfusion injury in rat. Nephrol Dial Transplant 23: 91-100, 2008.

23. Hou S, Ding H, Lv Q, Yin X, Song J, Landén NX and Fan H: Therapeutic effect of intravenous infusion of perfluorocarbon emulsion on LPS-induced acute lung injury in rats. PLoS One 9: e87826, 2014.

24. Collins T, Read MA, Neish AS, Whitley MZ, Thanos D and Maniatis T: Transcriptional regulation of endothelial cell adhesion molecules: NF-kappa B and cytokine-inducible enhancers. FASEB J 9: 899-909, 1995.

25. Min JK, Kim YM, Kim SW, Kwon MC, Kong YY, Hwang IK, Won MH, Rho J and Kwon YG: TNF-related activation-induced cytokine enhances leukocyte adhesiveness: Induction of ICAM-1 and VCAM-1 via TNF receptor-associated factor and protein kinase C-dependent NF-kappaB activation in endothelial cells. J Immunol 175: 531-40, 2005.

26. Li HL, Chen HL, Li H, Zhang KL, Chen XY, Wang XW, Kong QY and Liu J: Regulatory effects of emodin on NF-kappaB activation and inflammatory cytokine expression in RAW 264.7 macrophages. Int J Mol Med 16: 41-47, 2005.
27. Lawrence T: The nuclear factor NF-kappaB pathway in inflammation. Cold Spring Harb Perspect Biol 1: a001651, 2009.

28. Yu R, Kim CS, Kawada T, Kwon TW, Lim TH, Kim YW and Kwon BS: Involvement of leukotactin-1, a novel CC chemokine, in human atherosclerosis. Atherosclerosis 174: 35-42, 2004.

29. Jung J, Ko SH, Yoo do Y, Lee JY, Kim YJ, Choi SM, Kang KK, Yoon HJ, Kim H, Youn J and Kim JM: 5,7-Dihydroxy-3,4,6-trimethoxyflavone inhibits intercellular adhesion molecule 1 and vascular cell adhesion molecule 1 via the Akt and nuclear factor- $\kappa \mathrm{B}$-dependent pathway, leading to suppression of adhesion of monocytes and eosinophils to bronchial epithelial cells. Immunology 137: 98-113, 2012.

30. Zhu HH and Jiang LL: Serum inter-cellular adhesion molecule 1 is an early marker of diagnosis and prediction of severe acute pancreatitis. World J Gastroenterol 18: 2554-2560, 2012.

31. Brown MD and Sacks DB: Protein scaffolds in MAP kinase signalling. Cell Signal 21: 462-469, 2009.

32. Wu L, Cai B, Zheng S, Liu X, Cai H and Li H: Effect of emdoin on endoplasmic reticulum stress in rats with severe acute pancreatitis. Inflammation 36: 1020-1029, 2013.

33. Rani N, Bharti S, Bhatia J, Tomar A, Nag TC, Ray R and Arya DS: Inhibition of TGF- $\beta$ by a novel PPAR-Y agonist, chrysin, salvages $\beta$-receptor stimulated myocardial injury in rats through MAPKs-dependent mechanism. Nutr Metab (Lond) 12: 11, 2015.

34. Wu F, Wang H, Li J, Liang J and Ma S: Homoplantaginin modulates insulin sensitivity in endothelial cells by inhibiting inflammation. Biol Pharm Bull 35: 1171-1177, 2012.

35. Liu T, Fei Z, Gangavarapu KJ, Agbenowu S, Bhushan A, Lai JC, Daniels CK and Cao S: Interleukin-6 and JAK2/STAT3 signaling mediate the reversion of dexamethasone resistance after dexamethasone withdrawal in 7TD1 multiple myeloma cells. Leuk Res 37: 1322-1328, 2013.

36. Castillo RL, Carrasco Loza R and Romero-Dapueto C: Pathophysiological approaches of acute respiratory distress syndrome: Novel bases for study of lung injury. Open Respir Med J 9: 83-91, 2015.

37. Choi JS, Choi YJ, Park SH, Kang JS and Kang YH: Flavones mitigate tumor necrosis factor-alpha-induced adhesion molecule upregulation in cultured human endothelial cells: Role of nuclear factor-kappa B. J Nutr 134: 1013-1019, 2004.

38. Madan B, Batra S and Ghosh B: 2'-hydroxychalcone inhibits nuclear factor-kappaB and blocks tumor necrosis factor-alphaand lipopolysaccharide-induced adhesion of neutrophils to human umbilical vein endothelial cells. Mol Pharmacol 58: 526-534, 2000.

39. Ramudo L, Yubero S, Manso MA, Sanchez-Recio J, Weruaga E and De Dios I: Effects of dexamethasone on intercellular adhesion molecule 1 expression and inflammatory response in necrotizing acute pancreatitis in rats. Pancreas 39: 1057-1063, 2010. 